\title{
水系钠离子电池普鲁士蓝正极材料的制备与电化学性能研究
}

\author{
李 勇 $^{1}$, 何玮金鉷 ${ }^{1}$, 郑芯月 ${ }^{1}$, 于胜兰 ${ }^{2}$, 李海同 ${ }^{1}$, \\ 黎弘毅 ${ }^{1}$, 张 蓉 $^{1}$, 王 雨 $^{1}$
}

(1. 南昌大学 材料科学与工程学院, 南昌 330031; 2. 东华理工大学 化学生物与材料科学学院, 南昌 330013)

摘 要: 普鲁士蓝(PB)是一种金属有机骨架配合物, 作为正极材料在水系钠离子电池中有广泛的应用前景。本文采 用单一源法制备 PB, 系统研究了反应温度、反应时间以及盐酸浓度对 PB 形貌结构和电化学性能的影响。研究结 果表明, 升高反应温度能提高 PB 结晶性和循环稳定性, 以 $80{ }^{\circ} \mathrm{C}$ 合成的 $\mathrm{PB}$ 为正极材料组装的电池在 100 圈充放电 循环后容量保持率为 $93.9 \%$ 。延长反应时间可以使 $\mathrm{PB}$ 粒径增大，但是反应时间超过 $6 \mathrm{~h}$ 后 $\mathrm{PB}$ 粒径基本保持不变。 延长反应时间有利于提高循环性能, $10 \mathrm{~h}$ 所合成 PB 组装的电池在 100 圈充放电循环后容量保持率可以达到 $90 \%$ 。 提高盐酸浓度会改变 PB 的表面形貌, 同时改善电化学性能。盐酸浓度为 $0.20 \mathrm{~mol} / \mathrm{L}$ 时, 所得 PB 组装的电池经过 100 个循环后, 比容量仍有 $67.5 \mathrm{mAh} / \mathrm{g}$ 。本研究可以为制备高性能 $\mathrm{PB}$ 基水系钠离子电池提供理论和实验指导。

关 键 词: 水系钠离子电池; 单一源法; 普鲁士蓝; 循环稳定性

中图分类号: TM912 文献标识码: A

\section{Prussian Blue Cathode Materials for Aqueous Sodium-ion Batteries: Preparation and Electrochemical Performance}

\author{
LI Yong ${ }^{1}$, HE Wei-Xin ${ }^{1}$, ZHENG Xin-Yue ${ }^{1}$, YU Sheng-Lan ${ }^{2}$, \\ LI Hai-Tong ${ }^{1}$, LI Hong-Yi ${ }^{1}$, ZHANG Rong ${ }^{1}$, WANG Yu ${ }^{1}$
}

(1. School of Materials Science and Engineering, Nanchang University, Nanchang 330031, China; 2. College of Chemistry, Biology and Material Science, East China University of Technology, Nanchang 330013, China)

\begin{abstract}
Prussian blue (PB) is a kind of metal-organic framework complex that displays wide application prospect as cathode material for aqueous sodium-ion batteries. In this study, PB composites were prepared by a single source method. Furthermore, effects of reaction temperature, time and concentration of hydrochloric acid on PB morphology and electrochemical performance were systematically investigated. The results showed that crystallinity and electrochemical stability of PB were improved by increasing reaction temperature. The aqueous sodium-ion battery with PB synthesized at $80{ }^{\circ} \mathrm{C}$ as cathode material displayed a capacity retention of $93.9 \%$ after 100 cycles. The particle size of PB grew with the extension of reaction time until $6 \mathrm{~h}$. It's exhibited that the extended reaction time was beneficial to the cycle performance of device fabricated with PB prepared for $10 \mathrm{~h}$, delivering $90 \%$ capacity retention after 100 cycles. Increment of the hydrochloric concentration acid changed the surface morphology, and thus improved electrochemical performance of PB. When the concentration of hydrochloric acid reached $0.20 \mathrm{~mol} / \mathrm{L}$, a capacity of $67.5 \mathrm{mAh} / \mathrm{g}$ could be maintainted after 100 discharge-charge. This work may provide theoretical and experimental gaidence for
\end{abstract}

收稿日期：2018-06-20; 收到修改稿日期：2018-08-23

基金项目：国家自然科学基金地区科学基金项目(51562026) National Natural Science Foundation of China (51562026)

作者简介：李 勇(1987-), 男, 讲师. E-mail: liyong1@ncu.edu.cn

通讯作者：王 雨, 教授. E-mail: wangyu@ncu.edu.cn 
preparing high performance PB-based aqueous sodium-ion batteries.

Key words: aqueous sodium-ion battery; single source method; Prussian blue; cycle performance

随着传统化石能源的日趋枯竭以及随之带来的 环境问题, 大力开发新能源被认为是实现人类社会 可持续发展的首要课题。然而新能源存在稳定性差 等缺点, 因此通过发展储能技术解决新能源的稳定 性问题越来越受到重视 ${ }^{[1]}$ 。在众多二次能源体系中, 钠离子电池由于钠的储量丰富、价格低廉而备受关 注 $^{[2-4]}$ 。其中水系钠离子电池由于设备简单以及安全 性能高, 更是受到众多研究者青睐 ${ }^{[5]}$ 。当前已开发 出多种钠离子电池的正极材料, 如钴酸钠 ${ }^{[6-8]}$ 、锰 酸 钠 ${ }^{[9-10]}$ 和 普鲁士蓝 ${ }^{[11]}$ 等。其 中普鲁士蓝 $\mathrm{M}_{x} \mathrm{~N}_{y}\left[\mathrm{Fe}(\mathrm{CN})_{6}\right]$ (Prussian Blue, PB) 是一种简单的金 属有机骨架配合物, 分子式中 $M$ 为钠、钾等碱金属 元素, $N$ 则是过渡金属元素铁。由于 $P B$ 具有完整的 立方晶型结构, 且存在大量配位空隙 ${ }^{[11-15]}$, 有利于 碱金属离子在晶格中的嵌入与脱出; 同时得益于其 稳定的有机骨架，在碱金属离子的嵌入与脱嵌过程 中不会产生过大的形变, 所以 $\mathrm{PB}$ 在钠离子电池领 域引起了广泛关注。然而, 在制备 PB 过程中所形成 的晶体结构如空位缺陷、间隙水以及微观形貌等, 均会影响其电化学性能。因此, 研究 PB 的合成规律 和电化学性能的影响因素具有重要意义。

$\mathrm{PB}$ 的合成方法有电化学沉积法、水热法、模 板合成法、共沉淀法和单一源法等 ${ }^{[16]}$ 。其中单一源 法只需单一前驱体, 实验操作简单, 实验过程容易 控制, 且生成的 PB 颗粒尺寸均匀性较好。因此采 用单一源法制备 PB, 主要探究反应温度、反应时 间、盐酸浓度对 PB 的微观形貌结构和电化学性能 的影响。

\section{1 实验方法}

\section{1 实验原料}

十水合亚铁氧化钠 $\left(\mathrm{Na}_{4} \mathrm{Fe}(\mathrm{CN})_{6} \cdot 10 \mathrm{H}_{2} \mathrm{O}, \mathrm{AR}\right.$, 上 海阿拉丁生化科技股份有限公司), 氯化钠 $(\mathrm{NaCl}$, $\mathrm{AR}$, 上海阿拉丁生化科技股份有限公司), 抗坏血 酸 $\left(\mathrm{C}_{6} \mathrm{H}_{8} \mathrm{O}_{6}, \mathrm{VC}, \mathrm{AR}\right.$ ，上海阿拉丁生化科技股份有限 公司), 无水硫酸钠 $\left(\mathrm{Na}_{2} \mathrm{SO}_{4}, \mathrm{AR}\right.$, 上海阿拉丁生化 科技股份有限公司), 科琴黑(KB, EC600JD, Ketjenblack International)，浓盐酸 $(\mathrm{HCl}, 37 \mathrm{wt} \%, \mathrm{GR}$ ，西陇 科学股份有限公司), 聚偏氟乙烯 $\left(\left(\mathrm{C}_{2} \mathrm{H}_{2} \mathrm{~F}_{2}\right)_{x}, \mathrm{PVDF}\right.$, 电池级, 广州市南屋电子商务责任有限公司), $N$-甲 基吡咯烷酮 $\left(\mathrm{C}_{5} \mathrm{H}_{9} \mathrm{NO}, \mathrm{NMP}, \mathrm{AR}\right.$, 天津市大茂化学试
剂厂), 钛箔(电池级, 仁信金属材料有限公司)。

\section{2 制备普鲁士蓝}

采用单一源法制备 $\mathrm{PB}$ 并原位复合 $\mathrm{KB}$ 。将去离 子水加热到 $80{ }^{\circ} \mathrm{C}$, 并快速用该热水溶剂制备 $100 \mathrm{~mL} 20 \mathrm{mmol} / \mathrm{L}$ 的 $\mathrm{Na}_{4} \mathrm{Fe}(\mathrm{CN})_{6}$ 溶液, 再加入 $48 \mathrm{mg} \mathrm{KB}$, 超声 $15 \mathrm{~min}$, 使 $\mathrm{KB}$ 均匀分散在 $\mathrm{Na}_{4} \mathrm{Fe}(\mathrm{CN})_{6}$ 溶液中, 超声后在溶液中加入 $14.5 \mathrm{~g}$ $\mathrm{NaCl}$ 和 $0.088 \mathrm{~g}$ 还原剂 $\mathrm{VC}$, 将上述溶液放入水浴 锅中, 待温度上升到反应温度加入一定量的 $\mathrm{HCl}$, 持续搅拌, 反应结束后得到沉淀物, 用去离子水离 心洗涤 3 5 次, 转速 $10000 \mathrm{r} / \mathrm{min}$, 时间 $3 \mathrm{~min}$, 直至 离心后的上层清液 $\mathrm{pH}=7,80{ }^{\circ} \mathrm{C}$ 真空烘干 $24 \mathrm{~h}$ 后得到 目标产物。控制反应时间为 $4 \mathrm{~h}$ 、盐酸浓度为 $0.10 \mathrm{~mol} / \mathrm{L}$ 不变，通过改变反应温度 $\left(50 、 60 、 65 、 70\right.$ 或 $\left.80{ }^{\circ} \mathrm{C}\right)$ 探究温度的影响, 样品分别标记为 $\mathrm{PB}-50^{\circ} \mathrm{C} 、 \mathrm{~PB}-$ $60^{\circ} \mathrm{C} 、 \mathrm{~PB}-65^{\circ} \mathrm{C} 、 \mathrm{~PB}-70^{\circ} \mathrm{C}$ 和 $\mathrm{PB}-80^{\circ} \mathrm{C}$; 控制反应温 度为 $65{ }^{\circ} \mathrm{C}$ 、盐酸浓度为 $0.10 \mathrm{~mol} / \mathrm{L}$ 不变, 研究反应 时间(4、6、8 或 $10 \mathrm{~h}$ )的影响, 样品分别标记为 PB$4 \mathrm{~h} 、 \mathrm{~PB}-6 \mathrm{~h} 、 \mathrm{~PB}-8 \mathrm{~h}$ 和 PB-10h; 控制反应温度为 $65^{\circ} \mathrm{C}$ 、 反应时间为 $4 \mathrm{~h}$ 不变, 研究盐酸浓度 $(0.05 、 0.10 、 0.15$ 或 $0.20 \mathrm{~mol} / \mathrm{L}$ ) 的影响, 样品分别标记为 PB- $0.05 \mathrm{M}$ 、 PB-0.10M、PB-0.15M 和 PB-0.20M。

\section{3 电池制备}

将 1.2 制得的样品作为活性物质。先称取 $10 \mathrm{mg}$ 粘结剂 PVDF, 加入适量的溶剂 NMP 配成 $3 \mathrm{wt} \%$ 的 胶体, 再加入 $90 \mathrm{mg}$ 充分研磨过的活性物质并搅拌 均匀制得浆料。此后把制得的浆料均匀涂敷在钛箔 上, 然后放入 $60{ }^{\circ} \mathrm{C}$ 烘箱烘 $2 \mathrm{~h}$, 再在 $90{ }^{\circ} \mathrm{C}$ 的真空干燥 箱中真空干燥 $10 \mathrm{~h}$, 取出后称重待用。采用 $1 \mathrm{~mol} / \mathrm{L}$ 的 $\mathrm{Na}_{2} \mathrm{SO}_{4}$ 溶液作为电解液, 普鲁士蓝电极材料(普 鲁士蓝载量: $2 \mathrm{mg} \cdot \mathrm{cm}^{-2}$ ) 作为工作电极和对电极, 以 饱和甘录电极作为参比电极构成完整的水系电池测 试体系。以 $\mathrm{PB}-50^{\circ} \mathrm{C} 、 \mathrm{~PB}-60^{\circ} \mathrm{C} 、 \mathrm{~PB}-65^{\circ} \mathrm{C} 、 \mathrm{~PB}-70^{\circ} \mathrm{C}$ 或 $\mathrm{PB}-80{ }^{\circ} \mathrm{C}$ 作为电极材料所得电池分别标记为 BPB- $500^{\circ} \mathrm{C} 、 \mathrm{BPB}-60{ }^{\circ} \mathrm{C} 、 \mathrm{BPB}-65^{\circ} \mathrm{C} 、 \mathrm{BPB}-70{ }^{\circ} \mathrm{C}$ 和 BPB- $80^{\circ} \mathrm{C}$; 类似地, 采用 $\mathrm{BPB}-4 \mathrm{~h} 、 \mathrm{BPB}-6 \mathrm{~h} 、 \mathrm{BPB}-8 \mathrm{~h}$ 、 BPB-10h、BPB-0.05M、BPB-0.10M、BPB-0.15M 和 BPB-0.20M 分别指代以 $\mathrm{PB}-4 \mathrm{~h}$ 、 $\mathrm{PB}-6 \mathrm{~h}$ 、 $\mathrm{PB}-8 \mathrm{~h}$ 、 PB-10h 、PB- $0.05 \mathrm{M} 、 \mathrm{~PB}-0.10 \mathrm{M} 、 \mathrm{~PB}-0.15 \mathrm{M}$ 或 PB- $0.20 \mathrm{M}$ 作为电极材料所得电池。 


\section{4 表征与测试}

采用 $\mathrm{X}$ 射线衍射仪(XRD, D8 ADVANCE, 德国 布鲁克公司)进行结构表征, $2 \theta=10^{\circ} \sim 90^{\circ}$ 。采用扫描 电子显微镜(SEM, JSM 6701F, 日本电子株式会社) 进行形貌表征。采用透射电子显微镜(TEM, JEM$2100, J E O L)$ 表征纳米级别材料的形貌结构。采用蓝 电电池测试系统(CT2001A, 武汉市蓝电电子股份 有限公司)进行电化学性能测试。

\section{2 结果与讨论}

\section{1 单一源法合成普鲁士蓝的机理}

前驱体 $\mathrm{Na}_{4} \mathrm{Fe}(\mathrm{CN})_{6}$ 在酸性氛围下, 部分 $\mathrm{Fe}(\mathrm{CN})_{6}{ }^{4-}$ 中的 $\mathrm{Fe}^{2+}$ 会分离出来, 并可能被氧化成 $\mathrm{Fe}^{3+}$ 。 $\mathrm{Fe}^{3+}$ 和 $\mathrm{Fe}^{2+}$ 都可与未分解的 $\mathrm{Fe}(\mathrm{CN})_{6}{ }^{4-}$ 结合, 生成 $\mathrm{PB}$ 沉 淀(图 1)。因此温度、时间和盐酸浓度等因素均会影 响 PB 的微观形貌和晶体结构, 进而改变 PB 的电化 学性能。

\section{2 反应温度的影响}

图 2 为不同反应温度下所合成 PB 的 XRD 图谱。 从 XRD 图谱可以看出, 不同温度条件下合成的样 品衍射峰相同, 说明合成的样品具有相同的面心立 方晶体结构 ${ }^{[11]}$ 。同时, PB- $50^{\circ} \mathrm{C}$ 的 XRD 衍射峰宽化 相对严重, 说明该样品晶粒小、结晶性差; 而其余样 品的衍射峰比较尖锐, 说明样品结晶性更好。总体 而言, 衍射峰随着反应温度的上升而更为尖锐, 说 明反应温度越高, PB 的结晶性越好。

图 3 为不同反应温度下所合成 PB 的 SEM 和 TEM
照片。 $\mathrm{PB}-50^{\circ} \mathrm{C}$ 晶粒结晶性差(图 3(a)); PB- $60^{\circ} \mathrm{C}$ 棱角 清晰分明, 颗粒分布在 $\mathrm{KB}$ 表面(图 3(b)); $\mathrm{PB}-65^{\circ} \mathrm{C}$ 分 散性较好(图 3(c)), 与此同时, 从 $\mathrm{PB}-65^{\circ} \mathrm{C}$ 的透射电镜 照片(图 3(f)) 可以看出 $\mathrm{PB}-65^{\circ} \mathrm{C}$ 晶粒的立方形貌清晰,

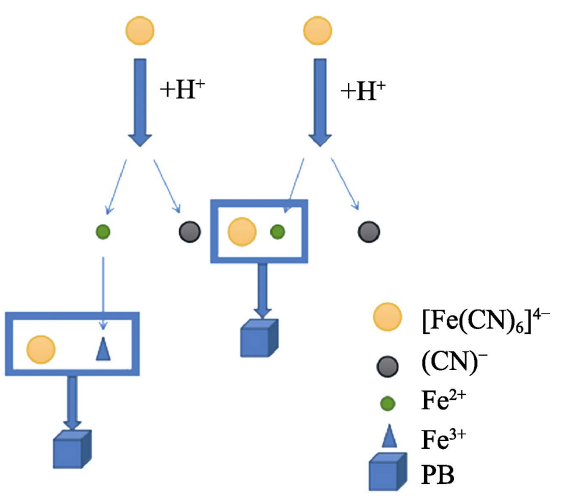

图 1 PB 沉淀形成机理图

Fig. 1 Diagram of PB precipitation mechanism

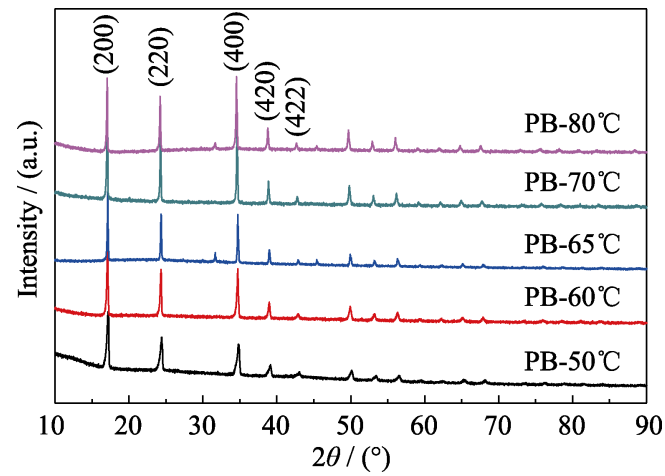

图 2 不同反应温度下所合成 PB 的 XRD 图谱

Fig. 2 XRD patterns of PB synthesized at different reaction temperatures
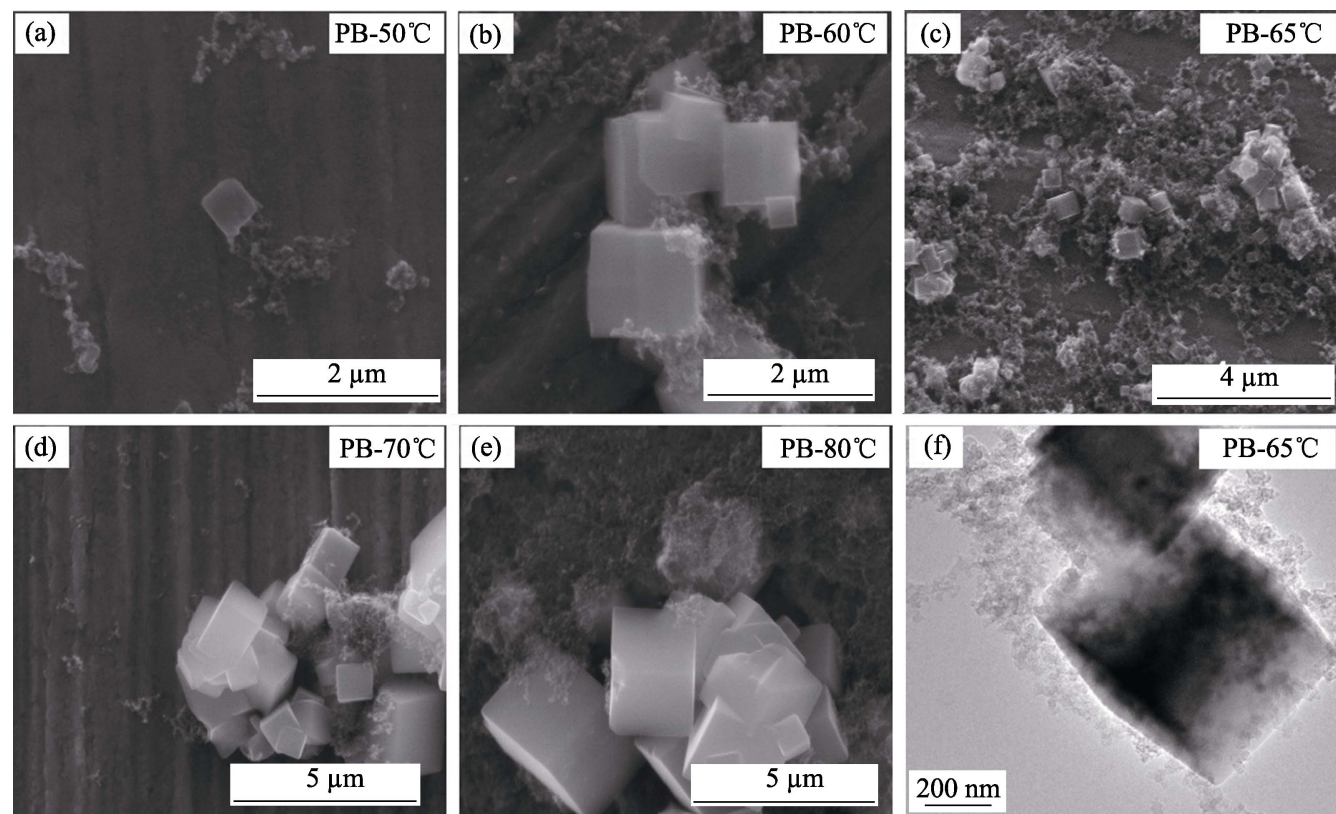

图 3 不同反应温度下所合成 PB 的(a) (e) SEM 和(f) TEM 照片

Fig. 3 (a-e) SEM and (f) TEM images of PB synthesized at different reaction temperatures 
棱角分明; $\mathrm{PB}-70^{\circ} \mathrm{C}$ 出现多层堆积现象, 颗粒较小的 $\mathrm{PB}$ 大多出现在较低一层 PB 的边角处(图 3(d)); PB$80^{\circ} \mathrm{C}$ 堆积现象更加明显(图 3(e))。从产率曲线(图 4) 可以看出 $\mathrm{PB}$ 产率随反应温度升高而升高, 其中 $\mathrm{PB}-$ $80^{\circ} \mathrm{C}$ 产率为 $96.5 \%$ 。 $\mathrm{KB}$ 可以为 $\mathrm{PB}$ 晶粒的成核提供 形核位点 ${ }^{[16]}$, 温度较低 $\left(50{ }^{\circ} \mathrm{C}\right)$ 时, $\mathrm{PB}$ 在 $\mathrm{KB}$ 上的成 核速率较低, 导致形成的 $\mathrm{PB}$ 晶粒较少。当温度上升 至 $60{ }^{\circ} \mathrm{C}$ 及以上时, 有利于 $\mathrm{PB}$ 晶粒成核生长, 因此 形成的 PB 晶粒都具有完整的立方体形貌 ${ }^{177-18]}$ 。当 温度较高时, 先形成的 $\mathrm{PB}$ 晶粒也能提供形核位点 ${ }^{[19]}$, 这种形核位点更易出现在 $\mathrm{PB}$ 晶粒的边角处, 直观 表现为 PB 晶粒向上倾斜堆积, 且随着温度升高, PB 能提供更多的形核位点, 因此 PB 堆积现象加剧, 产 率也随之升高。

从 XRD 图谱和形貌照片可以看出, $\mathrm{PB}-50^{\circ} \mathrm{C}$ 结 晶性最差, $\mathrm{PB}-65^{\circ} \mathrm{C}$ 结晶性和分散性都较好, $\mathrm{PB}-80^{\circ} \mathrm{C}$ 结晶性最好但团聚现象严重, 因此本研究选用 BPB$50^{\circ} \mathrm{C}, \mathrm{BPB}-65^{\circ} \mathrm{C}, \mathrm{BPB}-80^{\circ} \mathrm{C}$ 进行电化学性能分析(图 5 图 7)。从充放电曲线(图 5)可以看出, $\mathrm{BPB}-50^{\circ} \mathrm{C}$, BPB- $65^{\circ} \mathrm{C}, \mathrm{BPB}-80^{\circ} \mathrm{C}$ 表现出不同的初始放电能力, 其放电比容量分别为 $63.9,99.9$ 和 $52.7 \mathrm{mAh} / \mathrm{g}$, 其中 $\mathrm{BPB}-50^{\circ} \mathrm{C} 、 \mathrm{BPB}-65^{\circ} \mathrm{C}$ 出现两个电压平台, 分别为高 电压平台 $(0.6 \sim 1.0 \mathrm{~V})$ 和低电压平台 $(0.0 \sim 0.2 \mathrm{~V})$, 因 此比容量较高; 而 $\mathrm{BPB}-80^{\circ} \mathrm{C}$ 没有出现高电压平台, 比容量较低。对不同样品进行循环性能测试(图 6), 发现 BPB- $80^{\circ} \mathrm{C}$ 具有优异的循环稳定性, 在 100 圈循 环后容量保持率达 $93.9 \%$, 而 $\mathrm{BPB}-65^{\circ} \mathrm{C}$ 的容量保持 率仅 $39.3 \%$ 。结合充放电曲线分析可知, BPB- $65^{\circ} \mathrm{C}$ 充放电时出现两个平台, 会有更多的 $\mathrm{Na}^{+}$嵌入脱出, 导致其晶体结构不稳定, 这也是 $\mathrm{BPB}-65^{\circ} \mathrm{C}$ 循环稳 定性较差的原因。通过倍率性能测试(图 7)可以发现, 不同的电池在进行高倍率充放电后, 进行小电流

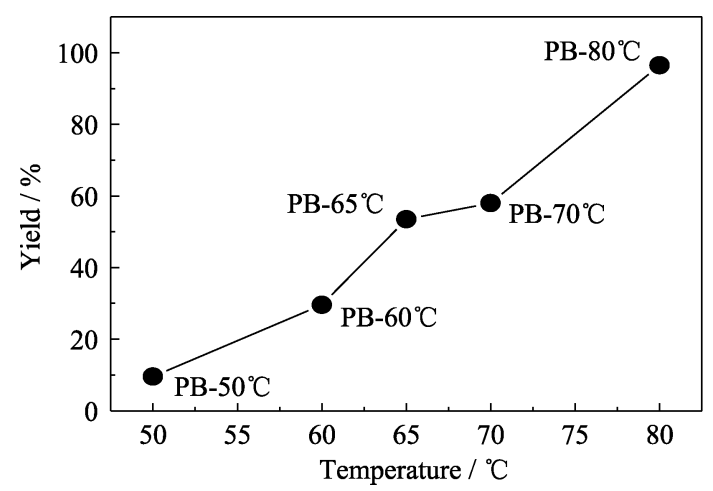

图 4 不同反应温度下所合成 PB 的产率曲线

Fig. 4 Yield curve of PB synthesized at different reaction temperatures
充放电依然能得到较高的比容量, 这说明在大电流 密度充放电过程中 PB 材料结构稳定性优异, 其中 $\mathrm{BPB}-65^{\circ} \mathrm{C}$ 具有更佳的倍率性能, 在 $1.0 \mathrm{~A} / \mathrm{g}$ 的充放 电电流密度下, 其比容量依然达到 $48.1 \mathrm{mAh} / \mathrm{g}$, 当 电流密度从 $1.0 \mathrm{~A} / \mathrm{g}$ 回到 $0.1 \mathrm{~A} / \mathrm{g}$ 时, 比容量保持率 达到 $93.1 \%$ 。

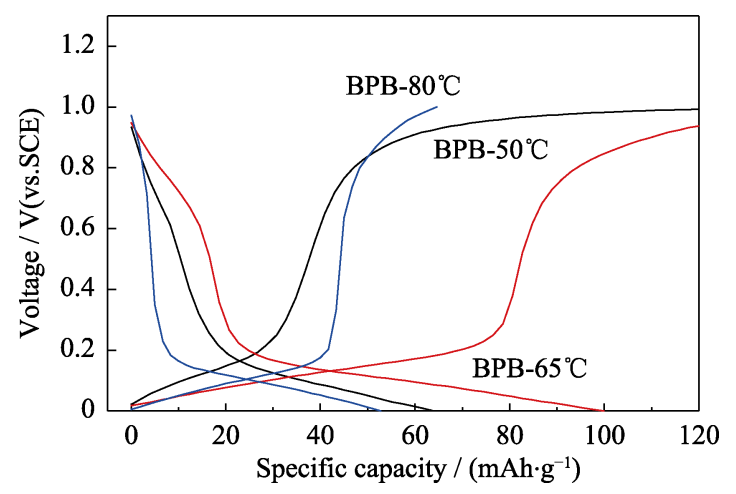

图 5 以不同反应温度下所得 $\mathrm{PB}$ 作为电极材料的电池的充 放电曲线

Fig. 5 Charge/discharge curves of batteries based on PB synthesized at different reaction temperatures

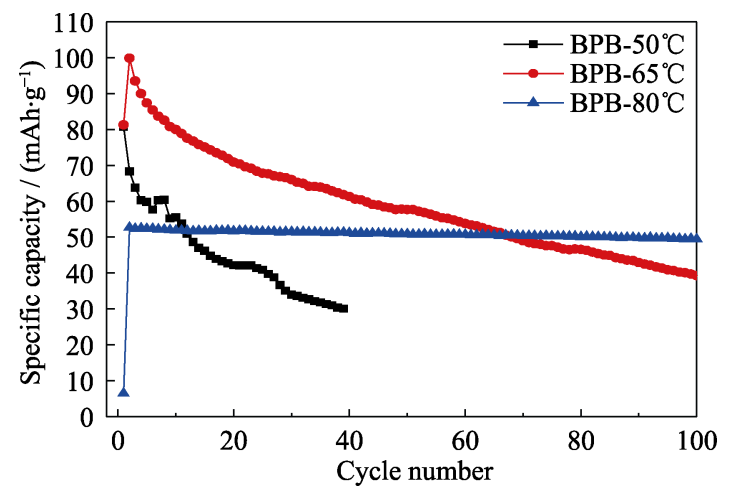

图 6 以不同反应温度下所得 $\mathrm{PB}$ 作为电极材料的电池的循 环性能

Fig. 6 Cycle performances of batteries based on PB synthesized at different reaction temperatures

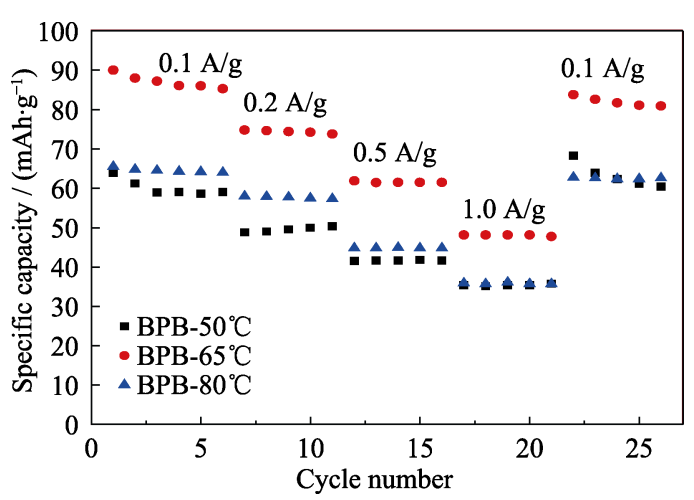

图 7 以不同反应温度下所得 $\mathrm{PB}$ 作为电极材料的电池的倍 率性能

Fig. 7 Rate performances of batteries based on PB synthesized at different reaction temperatures 


\section{3 反应时间的影响}

图 8 为不同反应下时间所合成 PB 的 XRD 图谱。 可以看出，样品都具有相同的 PB 特征峰。PB-4h 衍 射峰相对宽化, 表明该样品的晶粒较小。随着反应 时间延长，衍射峰越来越尖锐，说明通过延长反应 时间可以提高 PB 的结晶性。

图 9 为不同反应时间下所合成 PB 的 SEM 照片。 如图 9 所示, PB-4h 晶粒粒径为 $500 \mathrm{~nm}$ 左右, 堆积 现象不明显; 当时间达到 $6 \mathrm{~h}$ 或更长, 形成的 PB 晶 粒粒径范围为 $1 \sim 2 \mu \mathrm{m}$, 且都出现了堆积的现象; PB-4h 和 PB-6h 的 SEM 照片表明, PB 晶粒的粒径随 着反应时间的延长而增大。但是进一步延长反应时 间，PB 晶粒粒径不再增大，粒径曲线图可看出粒径 在 $6 \mathrm{~h}$ 后基本不变(图 10(a)), 这是由于随着 PB 晶粒 体积的增大, 晶粒的比表面积下降, 晶界能也随之 降低，导致 PB 晶粒更趋向于稳定，因此晶粒不再

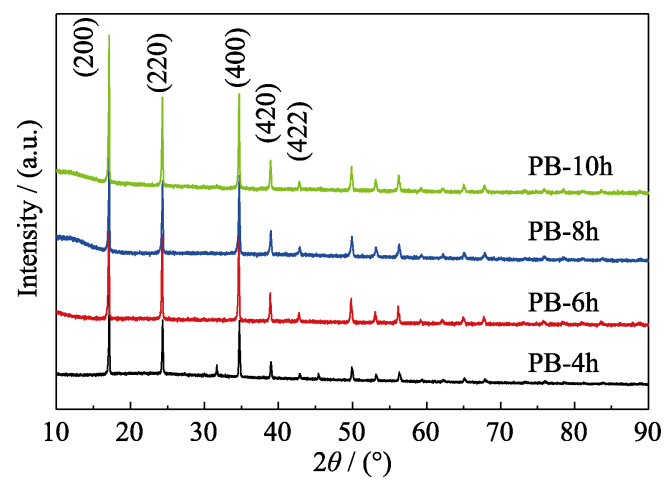

图 8 不同反应时间下所合成 $\mathrm{PB}$ 的 XRD 图谱

Fig. 8 XRD patterns of PB synthesized with different reaction time
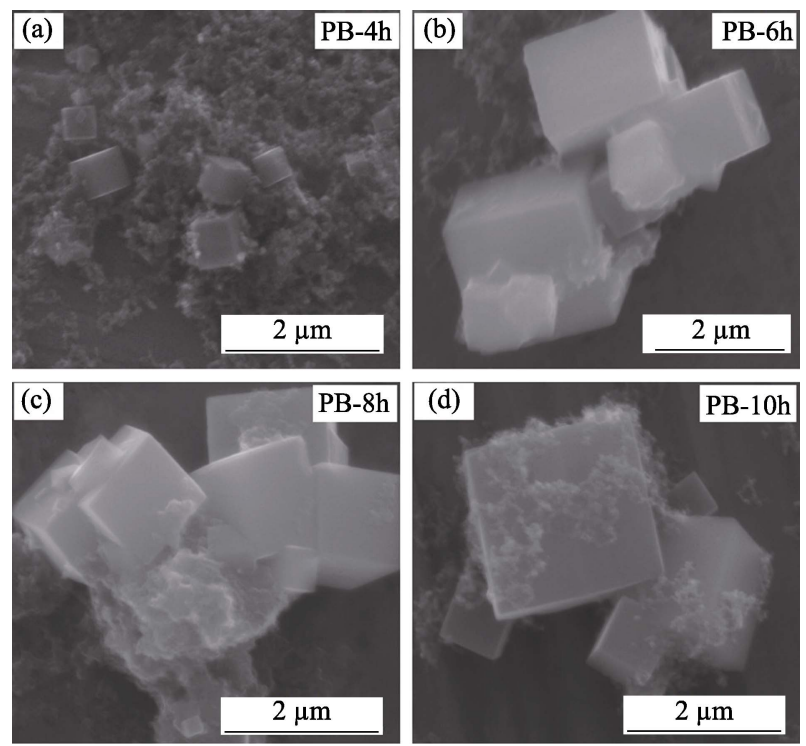

图 9 不同反应时间下所合成 PB 的 SEM 照片

Fig. 9 SEM images of PB synthesized with different reaction time
进一步长大 ${ }^{[20]}$ 。从图 10(b) 可以看出, PB-6h、PB- $8 \mathrm{~h}$ 、 $\mathrm{PB}-10 \mathrm{~h}$ 的产率相近，这是由于相同温度下，KB 和 $\mathrm{PB}$ 能提供的形核位点有限，延长反应时间不能促 进形核。

由于 PB-4h 粒径最小, 而 PB-10h 结晶性最好, 所以选用 BPB-4h 和 BPB-10h 进行电化学性能分析 (图 11 图 13)。BPB-4h 和 BPB-10h 的初始放电比容 量分别为 99.9 和 $61.2 \mathrm{mAh} / \mathrm{g}$ (图 11), BPB-4h 出现高 电压平台，表现出更高的比容量。而 BPB-10h 充放 电过程中只出现低电压平台, 所以在 $\mathrm{Na}^{+}$嵌入与脱 出过程中不容易引起结构性的破坏，因此循环
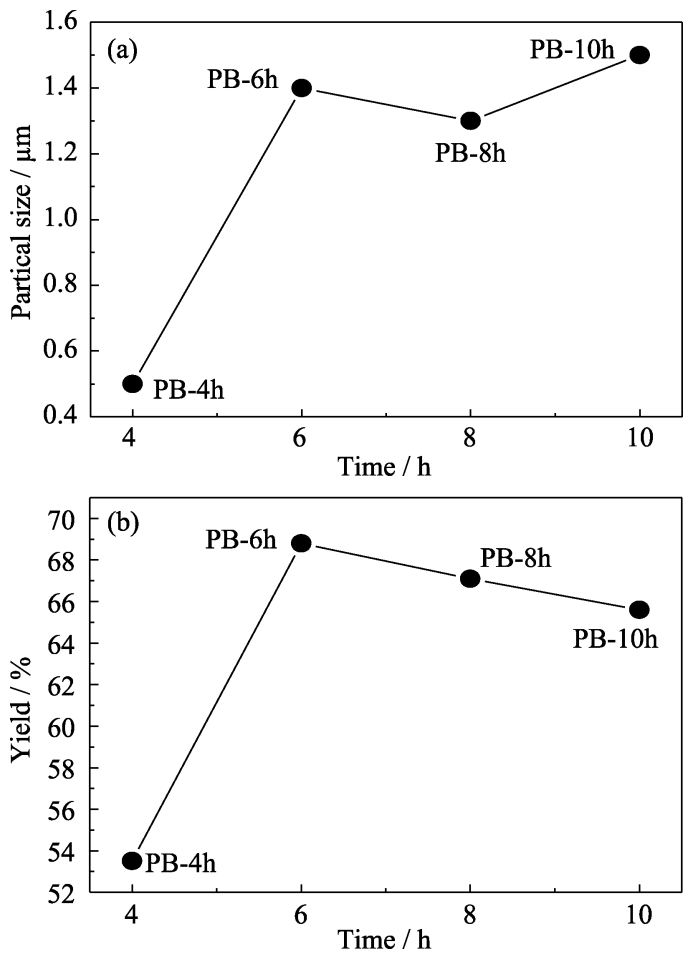

图 10 不同反应时间下所合成 PB 的(a)粒径曲线和(b)产率 曲线

Fig. 10 (a) Particle size and (b) yield curves of PB synthesized with different reaction time

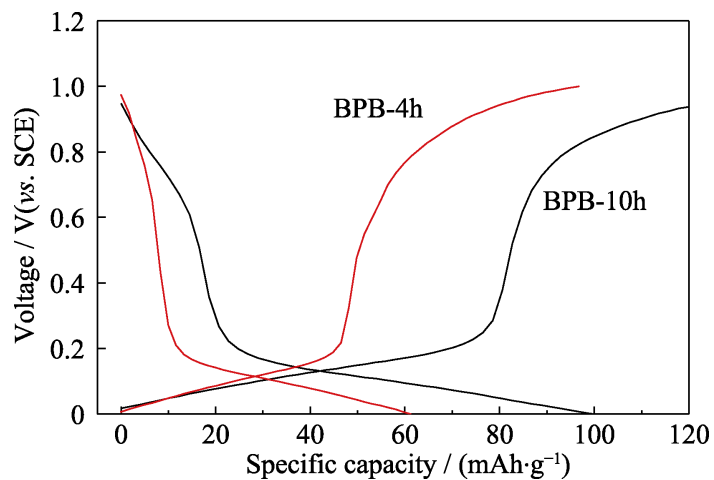

图 11 以不同反应时间下所得 $\mathrm{PB}$ 作为电极材料的电池的充 放电曲线

Fig. 11 Charge/discharge curves of batteries based on PB synthesized with different reaction time 


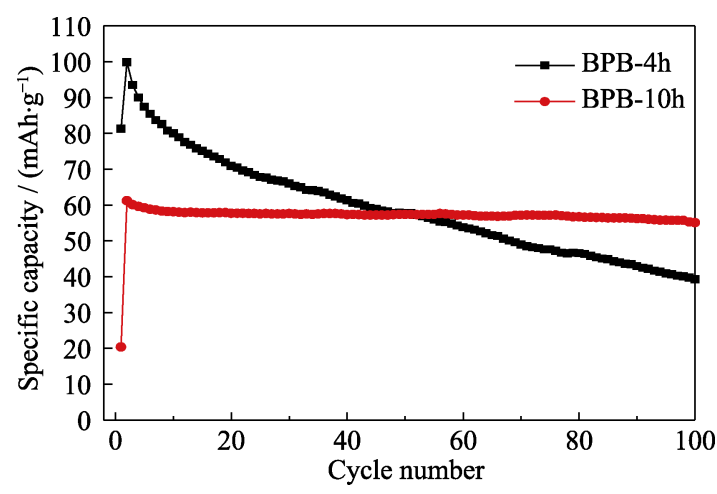

图 12 以不同反应时间下所得 PB 作为电极材料的电池的循 环性能

Fig. 12 Cycle performances of batteries based on PB synthesized with different reaction time

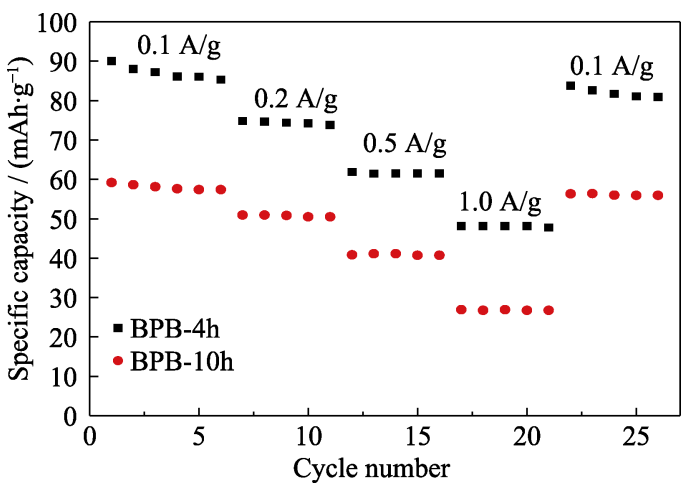

图 13 以不同反应时间下所得 PB 作为电极材料的电池的倍 率性能

Fig. 13 Rate performances of batteries based on PB synthesized with different reaction times

性能更优异, 其在 100 圈循环后容量保持率达到 90\%, 相比之下, BPB-4h 容量保持率仅有 $39.3 \%$ (图 12)。通过倍率性能测试(图 13)发现 BPB-10h 在 大电流密度充放电后回到 $0.1 \mathrm{~A} / \mathrm{g}$ 后依然具有 $56.3 \mathrm{mAh} / \mathrm{g}$ 的放电比容量, 保持率为 $95.1 \%$, 高于 BPB-4h 的 $93.1 \%$ 。

\section{4 盐酸浓度的影响}

图 14 为在不同盐酸浓度下所合成 PB 的 XRD 图谱。可以看出, 样品都具有相同的衍射峰, 说明都 形成了 $\mathrm{PB}$ 。不同反应条件会引起样品晶格常数的变 化, 从(200)晶面的衍射峰放大图可以看出, 随着盐 酸浓度提高, 衍射峰渐渐右移, 说明晶格常数变小。

图 15 为不同盐酸浓度下所合成 PB 的 SEM 照片。 PB-0.05M 和 PB- $0.10 \mathrm{M}$ 都具有完整的立方形貌, 晶 粒粒径在 $1 \mu \mathrm{m}$ 以下(图 15(a) (b)), 同时通过产率曲 线可以看出 PB-0.10M 的产率高于 PB-0.05M (图 16)。 而 PB-0.15M 和 PB-0.20M 的晶粒形貌不规则(图 $15(\mathrm{c}) \sim(\mathrm{d}))$ 。这是由于在酸性氛围下, $\mathrm{Fe}(\mathrm{CN})_{6}{ }^{4-}$

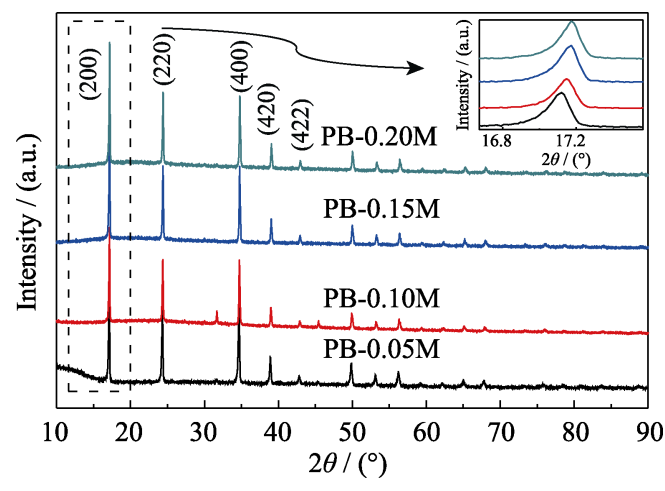

图 14 不同盐酸浓度下所合成 PB 的 XRD 图谱

Fig. 14 XRD patterns of PB synthesized at different concentrations of hydrochloric acid
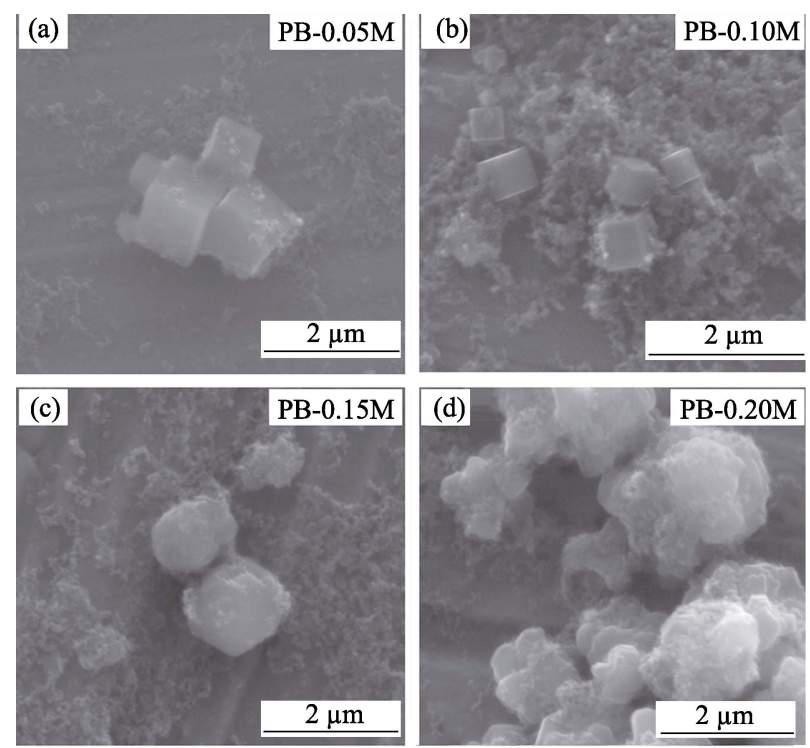

图 15 不同盐酸浓度下所合成 PB 的 SEM 照片

Fig. 15 SEM images of PB synthesized at different concentrations of hydrochloric acid

会分离出 $\mathrm{Fe}^{2+}$, 因此适当提高盐酸浓度有利于 $\mathrm{Fe}^{2+}$ 的分离, 从而提高 $\mathrm{PB}$ 的产率。但是盐酸浓度过高, 会腐蚀溶液中形成的 PB, 导致 PB 失去原有的立方 体形貌。

从 SEM 照片可以看出, PB- $0.10 \mathrm{M}$ 颗粒大少分 布较为均匀, 而 PB- $0.20 \mathrm{M}$ 腐蚀最严重, 因此本研究 选用 BPB-0.10M 和 BPB-0.20M 进行电化学性能分 析(图 17 图 19)。从充放电曲线(图 17)可以看出 BPB$0.10 \mathrm{M}$ 和 BPB- $0.20 \mathrm{M}$ 初始放电比容量相近, 分别为 99.9 和 $99.0 \mathrm{mAh} / \mathrm{g}$, 两者的电化学行为类似。通过 循环性能测试(图 18)可以看出, BPB-0.10M 在 100 圈循 环后，放电比容量只有 $39.3 \mathrm{mAh} / \mathrm{g}$, 而 BPB- $0.20 \mathrm{M}$ 仍 然保持 $67.5 \mathrm{mAh} / \mathrm{g}$ 的放电比容量, 容量保持率为 $68.2 \%$ 。因此，相比于 BPB-0.10M, BPB- $0.20 \mathrm{M}$ 的循 环稳定性得到了明显的改善, 这是由于腐蚀后 PB 的 $\mathrm{Na}^{+}$扩散路径更短, 使得 $\mathrm{Na}^{+}$的嵌入脱出过程更加 


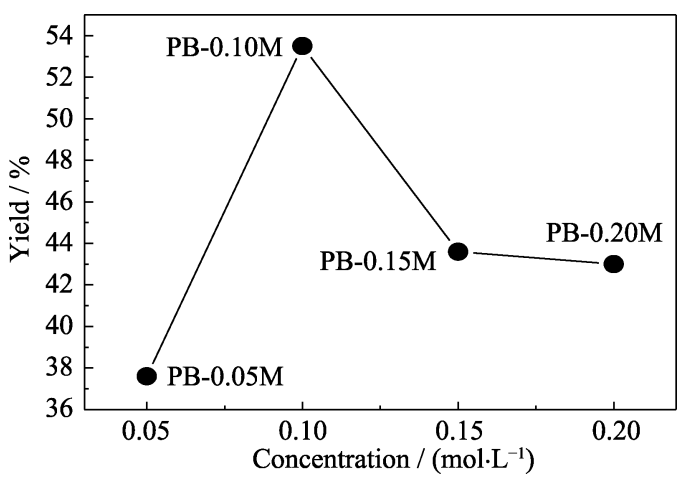

图 16 不同盐酸浓度下所合成 $\mathrm{PB}$ 的产率曲线

Fig. 16 Yield curve of PB synthesized at different concentrations of hydrochloric acid

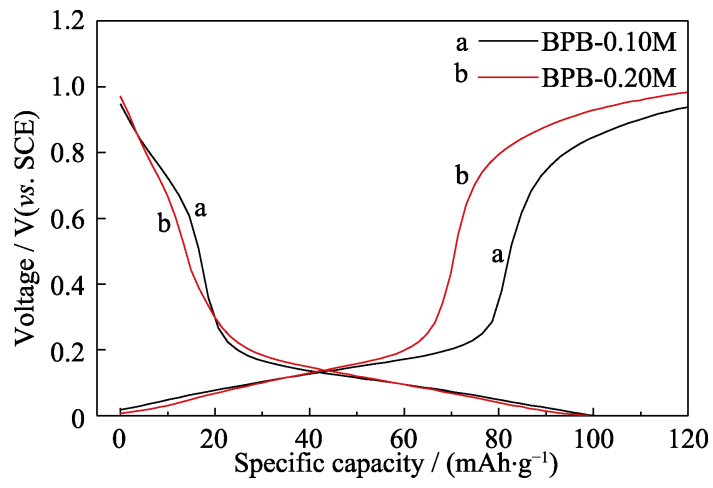

图 17 以不同盐酸浓度下所得 $\mathrm{PB}$ 作为电极材料的电池的充 放电曲线

Fig. 17 Charge/discharge curves of batteries based on PB synthesized at different concentrations of hydrochloric acid

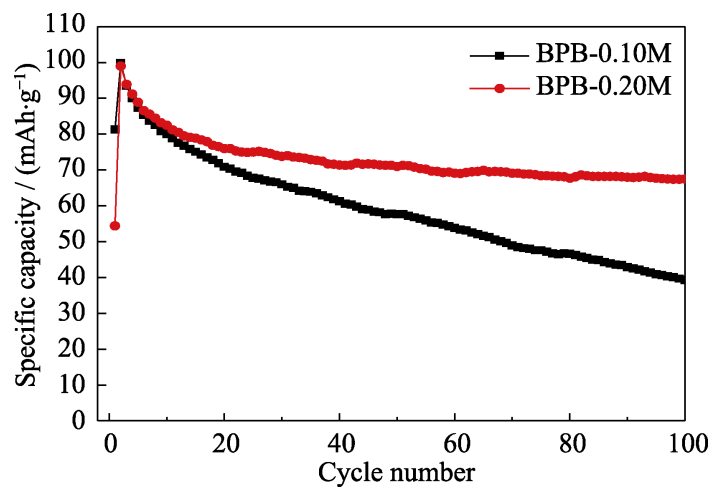

图 18 以不同盐酸浓度下所得 PB 作为电极材料的电池的循 环性能

Fig. 18 Cycle performances of batteries based on PB synthesized at different concentrations of hydrochloric acid

顺畅，因而改善了循环稳定性，这与文献[21]一致。 倍率性能测试(图 19)发现 BPB-0.20M 具有更加优异 的高倍率性能, 其在 $1.0 \mathrm{~A} / \mathrm{g}$ 的大电流密度充放电下 依然具有 $51.1 \mathrm{mAh} / \mathrm{g}$ 的比容量, 这可能是由于 PB$0.20 \mathrm{M}$ 因腐蚀而晶粒的尺寸缩小, 钠离子的嵌入脱 嵌路程缩短。

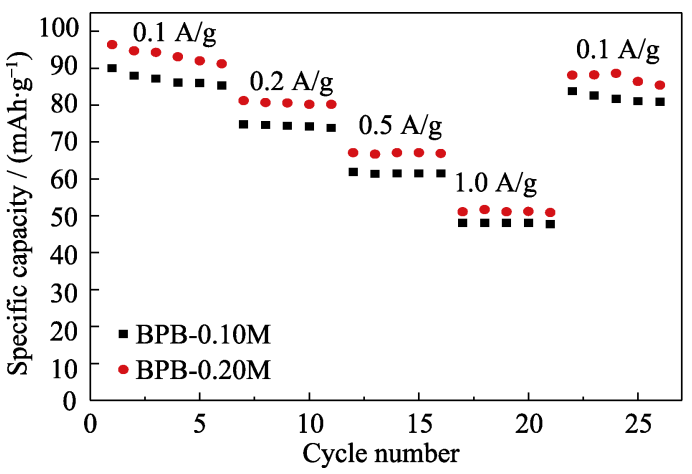

图 19 以不同盐酸浓度下所得 $\mathrm{PB}$ 作为电极材料的电池的倍 率性能

Fig. 19 Rate performances of batteries based on PB synthesized at different concentrations of hydrochloric acid

\section{3 结论}

普鲁士蓝( $\mathrm{PB}$ )在水系钠离子电池储能领域有广 泛的应用, 研究 PB 的合成规律以及电化学性能具 有重要意义。本文采用单一源法制备 PB 复合材料, 系统研究了反应温度、反应时间和盐酸浓度对 $\mathrm{PB}$ 形貌结构和电化学性能的影响, 研究发现:

1) 反应温度对 PB 的结晶性、堆积情况和产率 等都有影响，在 $50 \sim 80{ }^{\circ} \mathrm{C}$ 的条件下，随着温度上升， 形成的 $\mathrm{PB}$ 晶粒结晶性越来越好, 而堆积现象则越 来越严重, 产率也随之提高, 其中 $80{ }^{\circ} \mathrm{C}$ 条件下所合成 $\mathrm{PB}$ 的产率达到 $96.5 \%$ 。电化学性能测试表明, 以 $80{ }^{\circ} \mathrm{C}$ 合成的 PB 作正极材料组装的电池具有优异的循环 性能, 100 圈充放电循环后容量保持率达到 $93.9 \%$ 。 而 $65{ }^{\circ} \mathrm{C}$ 合成的 $\mathrm{PB}$ 组装的电池具有较好的倍率性能, 其在 $1.0 \mathrm{~A} / \mathrm{g}$ 的充放电电流密度下的放电比容量为 $48.1 \mathrm{mAh} / \mathrm{g}$ 。

2) 反应时间对 PB 晶粒的粒径有影响, $4 \mathrm{~h}$ 合成 的 PB 粒径在 $500 \mathrm{~nm}$ 左右, 粒径随着反应时间的延 长增大到 1 2 $\mu \mathrm{m}$, 但超过 $6 \mathrm{~h}$ 以后由于表面能降低, 晶粒不再进一步长大。10 h 合成的 PB 所组装的电 池展现出较好的循环性能, 在 100 圈充放电循环后 容量保持率达到 $90 \%$ 。

3) 盐酸浓度会改变 PB 的形貌和晶格常数, 在 盐酸浓度为 0.05 和 $0.10 \mathrm{~mol} / \mathrm{L}$ 的条件下, 不影响 $\mathrm{PB}$ 形貌，只影响产率，其中浓度为 $0.10 \mathrm{~mol} / \mathrm{L}$ 时产率 更高。而浓度为 0.15 和 $0.20 \mathrm{~mol} / \mathrm{L}$ 则会改变 $\mathrm{PB}$ 的 形貌, 其中浓度为 $0.20 \mathrm{~mol} / \mathrm{L}$ 可以明显改善 $\mathrm{PB}$ 作正 极材料所组装的电池的循环稳定性和倍率性能。

\section{参考文献:}

[1] WU X Y, LUO Y, SUN M Y, et al. Low-defect Prussian blue nanocubes as high capacity and long life cathode for aqueous 
Na-ion batteries. Nano Energy, 2015, 13: 117-123.

[2] YOU Y, YAO H R, XIN S, et al. Subzero-temperature cathode for a sodium-ion battery. Adv. Mater., 2016, 28(33): 7243-7248.

[3] CHEN Q, SUN S, ZHAI T, et al. Yolk-shell $\mathrm{NiS}_{2}$ nanoparticleembedded carbon fibers for flexible fiber-shaped sodium battery. Adv. Energ. Mater., 2018, 8(19): 1800054-1-9.

[4] GUO Q B, MA Y F, CHEN T T, et al. Cobalt sulfide quantum dot embedded N/S-doped carbon nanosheets with superior reversibility and rate capability for sodium-ion batteries. ACS Nano, 2017, 11(12): 12658-12667.

[5] CAI D P, YANG X H, QU B H, et al. Comparison of the electrochemical performance of iron hexacyanoferrate with high and low quality as cathode materials for aqueous sodium-ion batteries. Chem. Commun., 2017, 53(50): 6780-6783.

[6] D'ARIENZO M, RUFFO R, SCOTTI R, et al. Layered $\mathrm{Na}_{0.71} \mathrm{CoO}_{2}$ : a powerful candidate for viable and high performance Na-batteries. Phys. Chem. Chem. Phys., 2012, 14(17): 5945-5952.

[7] BERTHELOT R, CARLIER D, DELMAS C. Electrochemical investigation of the $\mathrm{P} 2-\mathrm{Na}_{x} \mathrm{CoO}_{2}$ phase diagram. Nature Mater, 2011, 10(1): 74-80.

[8] BHIDE A, HARIHARAN K. Physicochemical properties of $\mathrm{Na}_{x} \mathrm{CoO}_{2}$ as a cathode for solid state sodium battery. Solid State Ionics, 2011, 192(1): 360-363.

[9] CABALlERO A, HERNAN L, MORALES J, et al. Synthesis and characterization of high-temperature hexagonal $\mathrm{P} 2-\mathrm{Na}_{0.6} \mathrm{MnO}_{2}$ and its electrochemical behaviour as cathode in sodium cells. J. Mater. Chem., 2002, 12(4): 1142-1147.

[10] CAO Y L, XIAO L F, WANG W. et al. Reversible sodium-ion insertion in single crystalline manganese oxide nanowires with long cycle life. Adv. Mater., 2011, 23(28): 3155-3160.

[11] LU Y H, WANG L, CHENG J G, et al. Prussian blue: a new framework of electrode materials for sodium batteries. Chem.
Comm., 2012, 48(52): 6544-6546.

[12] HUANG Y X, XIE M, ZHANG J T, et al. A novel border-rich Prussian blue synthetized by inhibitor control as cathode for sodium ion batteries. Nano Energy, 2017, 39: 273-283.

[13] WANG B Q, HAN Y, CHEN Y T, et al. Gradient substitution: an intrinsic strategy towards high performance sodium storage in Prussian blue based cathodes. J. Mater. Chem. A, 2018, 6(19): 8947-8954.

[14] YOU Y, WU X L, YIN Y X, et al. High-quality Prussian blue crystals as superior cathode materials for room-temperature sodium-ion batteries. Energ. Environ. Sci., 2014, 7(5): 1643-1647.

[15] JIANG Y Z, YU S L, WANG B Q, et al. Prussian blue@C composite as an ultrahigh-rate and long-life sodium-ion battery cathode. Adv. Funct. Mater., 2016, 26(29): 5315-5321.

[16] 于胜兰. 钠离子电池正极材料普鲁士蓝类钠盐的制备及电化学 性能研究. 杭州: 浙江大学硕士学位论文, 2015.

[17] VAUCHER S, FIELDEN J, LI M, et al. Molecule-based magnetic nanoparticles: synthesis of cobalt hexacyanoferrate, cobalt pentacyanonitrosylferrate, and chromium hexacyanochromate coordination polymers in water-in-oil microemulsions. Nano Lett., 2002, 2(3): 225-229.

[18] ZHENG X J, KUANG Q, XU T, et al. Growth of Prussian blue microcubes under a hydrothermal condition: possible nonclassical crystallization by a mesoscale self-assembly. J. Phys. Chem. C, 2007, 111(12): 4499-4502.

[19] LIM B, JIANG M J, CAMARGO PHC, et al. Pd-Pt bimetallic nanodendrites with high activity for oxygen reduction. Science, 2009, 324(5932): 1302-1305.

[20] 胡鸣. 铁氧化物与普鲁士蓝类化合物的形貌可控合成与非经典 结晶机理研究. 上海: 华东师范大学博士学位论文, 2010.

[21] REN W H, QIN M S, ZHU Z X, et al. Activation of sodium storage sites in Prussian blue analogues via surface etching. Nano Lett., 2017, 17(8): 4713-4718. 